University of Wollongong

Research Online

SMART Infrastructure Facility - Papers

Faculty of Engineering and Information

Sciences

2016

Case Study: A Model Based Systems Engineering (MBSE) Framework for Characterising Transportation Systems Over the Full Life Cycle

William R. Scott

University of Wollongong, wscott@uow.edu.au

Richard Fullalove

Transport for New South Wales

Gary Arabian

Transport for New South Wales

Allan P. Campbell

University of Wollongong, pcampbel@uow.edu.au

Follow this and additional works at: https://ro.uow.edu.au/smartpapers

Part of the Engineering Commons, and the Physical Sciences and Mathematics Commons

Research Online is the open access institutional repository for the University of Wollongong. For further information contact the UOW Library: research-pubs@uow.edu.au 


\title{
Case Study: A Model Based Systems Engineering (MBSE) Framework for Characterising Transportation Systems Over the Full Life Cycle
}

\author{
Abstract \\ Development of transport infrastructure has significant challenges including acquisition lag, phased \\ evolution, multiple disparate stakeholders and environment-specific issues. The Asset Standards \\ Authority (ASA) at TfNSW is introducing MBSE to address these challenges. Instrumental to the \\ introduction is the development of a framework that structures the available data and provides guidance \\ and traceability between the data sets. This framework combines the enterprise-level drivers with the \\ lower level drivers such as standards to outline a generic conceptual design for a transport system. This \\ conceptual design is then used to guide future system development. The outcome is future project \\ information based on greater, enduring understanding of the transport system.

\section{Keywords} \\ systems, model, study:, case, full, transportation, characterising, framework, (mbse), engineering, over, \\ cycle, life \\ Disciplines \\ Engineering | Physical Sciences and Mathematics

\section{Publication Details} \\ Scott, W., Fullalove, R., Arabian, G. \& Campbell, P. (2016). Case Study: A Model Based Systems Engineering \\ (MBSE) Framework for Characterising Transportation Systems Over the Full Life Cycle. INCOSE \\ International Symposium, 26 (1), 916-932.
}




\title{
Case Study: A Model Based Systems Engineering (MBSE) Framework for Characterising Transportation Systems Over the Full Life Cycle
}

\author{
William Scott \\ SMART Infrastructure Facility \\ University of Wollongong NSW 2522 \\ Australia \\ $+61411892491$ \\ wscott@uow.edu.au
}

Richard Fullalove

Freight, Strategy and Planning Division

Transport for New South Wales

Australia

+61449884671

Richard.Fullalove@transport.nsw.gov.au
Gary Arabian

Freight, Strategy and Planning Division

Transport for New South Wales

Australia

+61431329212

Gary.Arabian@transport.nsw.gov.au

Peter Campbell

SMART Infrastructure Facility

University of Wollongong NSW 2522

Australia

+61429080414

pcampbel@uow.edu.au

Copyright (C 2016 by Author Name. Published and used by INCOSE with permission.

\begin{abstract}
Development of transport infrastructure has significant challenges including acquisition lag, phased evolution, multiple disparate stakeholders and environment-specific issues. The Asset Standards Authority (ASA) at TfNSW is introducing MBSE to address these challenges. Instrumental to the introduction is the development of a framework that structures the available data and provides guidance and traceability between the data sets. This framework combines the enterprise-level drivers with the lower level drivers such as standards to outline a generic conceptual design for a transport system. This conceptual design is then used to guide future system development. The outcome is future project information based on greater, enduring understanding of the transport system.
\end{abstract}

\section{Introduction}

Infrastructure refers to the fundamental facilities and systems serving a country, city, or area, including the services and facilities necessary for its economy to function (Sullivan \& Sheffrin 2003). It typically characterizes technical structures such as transport systems (roads, bridges, tunnels, etc.), water supply, sewers, electrical grids, telecommunications, and so forth, and can be defined as "the physical components of interrelated systems providing commodities and services essential to enable, sustain, or enhance societal living conditions" (Fulmer \& Jeffrey 2009). According to the mentioned definition, infrastructure inherently is a set of interrelated systems (also known as a system of systems (SoS)) that aims to provide services to the public and where the Government is liable for the availability and quality of those services.

However, large scale infrastructure projects are becoming more difficult to successfully deliver. Infrastructure systems around the world are under pressure to meet increased levels of performance, reliability and sustainability. In order to be able to accomplish these goals it is necessary for the responsible organizations to clearly understand all the interrelationships that exist, both within its' 
own structure and within other organizations that may be responsible for delivery. Added to this complexity in the transport sector are initiatives to develop a better integrated transport system that recognizes the fact that commuters often engage several modes of transport to get to their destination which need to be supported. For example, Transport for New South Wales (TfNSW) has committed itself to the delivery of an integrated transport strategy that aims to better manage the multi-modal transport that many commuters utilize to get to their destinations (TfNSW 2012). This requires an unprecedented level of knowledge and control of these SoSs to manage the complexities of their operation, maintenance and evolution. There are several challenges that are common to these large scale infrastructure projects as well as in the defence and aerospace industries

Complex SoS. Transport systems and the organizations which deliver them can be considered to be collections of SoSs that have to work in conjunction to provide a delivery system which can provide the necessary quality of service. At the lowest level, each transport system can be considered an SoS where individual routes form independent systems that transport people and freight through different regions. A wider view can be taken of the transport system to include multi-modal transport. In the case of TfNSW, which is responsible for several modes of public transport, this means that the various sub-units within TfNSW must have the means to clearly and unambiguously work together. This case study includes a generalized depiction which includes the transfer between modes of transport to provide commuters a more seamless transition.

Significant Lag in Acquisition. Once an acquisition has been identified, it can take 10-15 years to fully acquire and put into service the new equipment and systems. This is a problem that Defense acquisition organizations around the world also experience which prompted them to adopt architecture frameworks (AFs) in an attempt to address their challenges, beginning with C4ISR in the 1990s (US DoD 2010)

Phased evolution of large parallel systems. Evolution can be the result of reorganization or the introduction of new equipment into the transport system. Organizations often attempt to improve by changing structure: however, effort is needed to ensure that necessary roles are assigned appropriately and not inadvertently lost. Also, reorganization can take a significant time as the people learn to understand the new organization and become accustomed to how it works. Introduction of new equipment has to be phased due to the size and complexity of the transport system in combination with the need not to compromise the safety of the system. These considerations result in a complex problem in tracking the configuration of a system at any stage to ensure that safety is not inadvertently compromised and to limit the impact on commuters.

Environment-specific Issues and Requirements. Infrastructure systems are distributed over a wide region which causes some very specific challenges in identification, capture and tracking of region-specific requirements and in communicating these to the effected parties. The AF enables the project proponents to understand and address the range of often conflicting issues that arise across local regional boundaries as well as the differing socio-economic views of stakeholders.

Multiple stakeholders with disparate needs. The responsibilities for a transport SoS are spread between different organizations, including in our case, not just TfNSW divisions (See Figure 5), but also the Public Private Partnership (PPP) operators, and Authorized Engineering Organizations (AEOs) which may be engaged to construct the project. Each system that comprises the transport SoS often has an individual organization that is responsible for the operation or possibly only part of the operation. For example, heavy rail operations are spilt between passenger and freight which need to share resources. To further complicate the situation, other organizations are responsible for outlining the system needs, timetabling or the procurement of system upgrades. This results in a complex group of stakeholders who own aspects of the acquiring system as well as the operating system and may have competing needs.

TfNSW is responsible for the evolution of the existing public transport system to ensure that it continues to meet the needs of the population. The Asset Standards Authority (ASA) within 
TfNSW is currently leading the introduction of Model-based Systems Engineering (MBSE) practice to better manage the evolution of the transport network. Therefore, TfNSW largely contains people who have yet to adopt MBSE techniques, though many see the benefit once introduced. ASA is introducing MBSE through the use of an AF populated using SysML in Magic Draw (referred to hereafter as the Transport Network Architecture Framework (TNAF)). The TNAF is intended initially to be used to act as a structure that will assist procurement through the depiction of the minimum standards to be met when specifying new systems to be acquired and integrated into the network. The first set of activities has focused on heavy rail procurement. However, this version has also been genericized to apply to other modes of transport as well as support understanding how commuters have to utilize multimodal transport. The TNAF ensures that all aspects of how the existing system will evolve with the introduction of changes are considered and a consistent approach is taken for similar acquisitions. There have already been numerous opportunities that have been identified where TfNSW can benefit through the use of the TNAF. Some of these include:

- Interface identification for future upgrade compliance.

- Conduit for data transfer between stakeholders.

- Greater assurance for risk and safety evaluations

- Change management

- Competency management

- Improved Asset lifecycle management

- Improved overall knowledge management

SMART at the University of Wollongong has been engaged by the ASA to help develop the TNAF and enhance the MBSE tool environment. This paper aims to provide an overview of the TNAF content combined with the underlying TNAF development drivers.

The paper next provides an overview of AFs which have been selected for use in this work. This is followed by a discussion of the design philosophy used and the objectives of the AF development. The paper then progresses into more detail as to how some of the modelling has been done first on the enterprise level and then examining the bottom level drivers derived from documentation and existing operations. This is followed by a discussion of the generic depiction of a transport system and how this can be used to understand the system evolution sufficiently to provide safety assurance during system changes and upgrades, after which the paper concludes.

\section{Overview of Architecture Frameworks}

ISO/IEC/IEEE 42010 defines an Architecture Framework as: "conventions and common practices for architecture description established within a specific domain or stakeholder community" (ISO 2011). Building upon the requirements for specifying architecture descriptions in IEEE 1471 (IEEE 2000), the draft standard also specifies requirements for architecture frameworks which may be summarized as follows: a framework must identify: a set of stakeholders, a set of their architecture concerns, and a set of viewpoints framing those concerns.

In developing the TNAF AF we have used the TRAK metamodel as a guide (Plum 2011), but in the current work are using UPDM expressed in SysML.

Arguably, the two most widely used military frameworks are the Department of Defence Architecture Framework (DoDAF) (US DOD 2010) in the USA and the Ministry of Defence (MOD) Architecture Framework (MODAF) in the UK (UK MoD 2013, Hause 2010). The architecture framework called "The Railway Architecture frameworK" (TRAK) (Plum 2012) was developed by the RSSB (Rail Safety and Standards Board) in the UK and was initially based on MoDAF. TRAK conforms to the standard for architecture description ISO/IEC/IEEE 42010 (ISO 2011) and is tied to the Systems Engineering life cycle defined in ISO/IEC 15288 (ISO/IEC 2002. The definition of TRAK is concerned with "the what" not "the how", so at any time there may be 
many implementations of a TRAK conceptual design (Plum 2010). This view is consistent with DoDAF and MoDAF as there was not any standard method for implementation of the architecture models created by them until the appearance of UPDM (Unified Profile for DoDAF and MoDAF). UPDM is a Standardized way of expressing DoDAF and MODAF artefacts using OMG UML (OMG 2010a) and OMG SysML (OMG 2010b), as well as OMG XMI which is a standard for interchange (Hause 2009). Standardization of model data and UML/SysML mapping means that both tool vendors and industry can provide models in a single format (Hause 2010). So, it is worth studying how the UPDM instructions can be employed to implement an architecture created based on the TRAK metamodel.

\section{Underlying Modelling Philosophy}

It was recognized that the framework could yield a large and extensive model that could become difficult to use unless carefully structured to reflect the viewpoints and views needed by the users involved in TfNSW projects. Therefore a modelling philosophy was enforced to address issues such as usability and the ability to easily find and convey pertinent information. (Hause, 2010) The philosophy has three parts:

\section{Separation of Data from Views}

This makes the distinction between the data within the framework and the views into this data that are available. The data area is dedicated to the objects identified as a part of the framework and the relationships between these objects. The data area is subdivided to co-locate like data so that users are guided towards the data to reduce the likelihood that similar or repeated entries are created. Views are then made separately from this data. An advantage in this separation is that views need not display all of the information and so can be filtered to ensure the appropriate message is conveyed (more on the philosophy surrounding these views follows).

This separation has made it far easier to find desired elements in the framework and has resulted in far less replication of data when multiple users are involved.

\section{Customised and Constrained View}

When constructing views, there are several aspects that were considered: the message, audience, presentation and the existence of other associated views that need to be linked to the view under construction. There are times where all of the available information can effectively just be dumped into a diagram which makes it difficult to clearly gather and understand the desired information. By focusing on access to the needed information, and the intended audience, non-pertinent information can be filtered out to help quickly convey the intent. This aspect also constrains the amount of information being presented. Large complex diagrams, while complete, can be difficult to follow therefore a particular message may be partitioned into a family of views that will guide the user to the relevant information for various activities. How a user navigates between these views and to other associated diagrams then has to be considered, which is heavily dependent on the type of user. While some users are comfortable using standards such as SysML to receive information, others prefer other representations. Therefore some diagrams may be depicted slightly differently to cater to different audiences even though the content is consistent. Within the domain of infrastructure concerns which ASA hopes to support with the TNAF a number of different users have been identified. These include ASA systems engineering, ASA standards setting, TfNSW Customer Experience Division, Sydney Trains, NSW Country Rail Network, and Approved Engineering Organisations. The degree of expertise in MBSE and the use of AFs across this group is very variable.

To help identify the information contained within a diagram a TNAF identification system has been established. Based on ASA's 'network standards numbering system' (ASA 2014) for published transport documents the TNAF identification system (See Figure 1) identifies the type of view 
(based on the originating standard); the mode being represented; the applied discipline; and some human readable text.

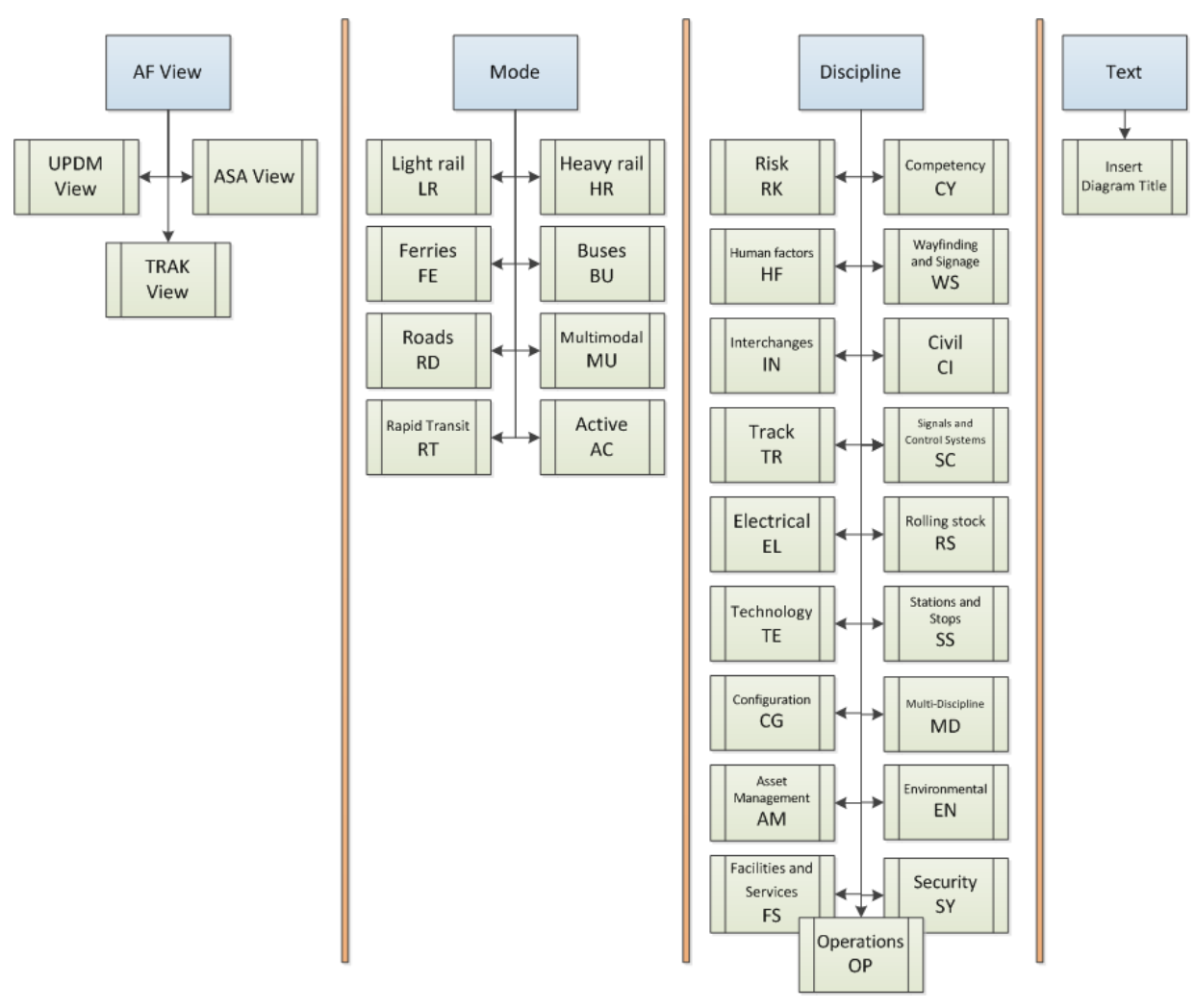

Figure 1: Overview of TNAF Identification System

\section{Framework Navigation through Views}

As described above derivation of views needs to be combined with the architecting of the navigation to allow the users to find the necessary information efficiently. Identification and prediction of common lines of inquiry first are needed to architect the view navigation. The links between the views can then be constructed to support the users moving through these lines of inquiry.

Framework navigation has also required the development of a small number of diagrams to act as an entry point into the framework and determine the appropriate initial view to begin a particular line of inquiry.

These three philosophies combine to improve the usability of the framework and increase the efficiency to be able to disseminate information.

\section{Objectives of the Framework for Characterising the Transportation System}

The development of the TNAF has been driven by several factors. The primary driver has been the ability to create a consistent view of the available information which includes the articulation of organization structures and interdependencies, the derivation of the desired capability from the organizational imperatives (i.e. top-down drivers), the impact of existing documents and standards (bottom up) and capture of the operational activities. The views provided by the TNAF are intended to be able to convey the required information within the context of the operation of the transport system. These views are then mapped onto the evolution of the system to understand how activities are to be undertaken both during transitions and once a system upgrade is complete. 
Another key need is to have the ability to capture the evolution of the system over time. Infrastructure projects generally have extended and phased implementation schedules, which can be subject to change from many sources and have to meet not only current and projected demands, but also integrate with other future infrastructure development that is under consideration. Therefore, knowledge is needed at a very detailed level of not only the system and its interfaces but how and when it will be deployed to ensure that the various system configurations are robust, safe and able to meet cost and schedule goals.

For the TNAF to meet the objectives of TfNSW there is a wide variety of information that has to be captured. At the higher level is the capture of the derivation of the capabilities and interdependencies from the goals and structures of the various organizations that comprise TfNSW. This organizational structure information includes the identification of roles assigned to divisions as well as to individual positions. As explained above, the initial work has largely focused on the heavy rail mode of transport; however, it is ASAs intention to expand the TNAF into other transport modes such as light rail, buses, ferries, walkways etc...As it currently stands, the TNAF also contains the generic design for a multi-modal transport system so any projects that are undertaken will become an element within an integrated transport network that spans from passenger departure to destination. This generic design is both physical and behavioral, enabling the specification of common elements that comprise the network and the associated behavior to be exhibited.

The TNAF has so far been decomposed down several levels to the point where operations implementation detail is necessary to depict the information more concisely. The conceptual model can be used to identify the necessary functionality and interfaces needed when procuring a system by mapping the project onto the conceptual model elements. Also, as indicated above, this conceptual design links the existing standards and developing documentation within the framework structure.

\section{Model Structure Overview}

A series of package diagrams have been developed to help understand the framework and to navigate through it. Figure 2 depicts the top level entry diagram that provides an overview of the areas within the framework. The framework is currently partitioned into three areas: organization, conceptual design and management. At the top can be seen the views with respect to the various organizations. There is a set of enterprise-specific views that provide entry for their stakeholders. There are also views that depict the goals of the various organizations, the goal interdependencies, and how goals are broken down into necessary capabilities (discussed in more detail below). The third element in the organisation area is the depiction of the organization structures that identify the posts with their associated roles.

Below the organization area is the conceptual design area. This is currently focused on depicting aspects of heavy rail however there is space reserved for other modes of transit. At the bottom of the diagram is the management area that contains the bottom-up drivers and related information. This area shows how metrics, common documents such as standards and reports as well as existing requirement specifications are captured so that they can be referenced and their implications flow through the framework. 


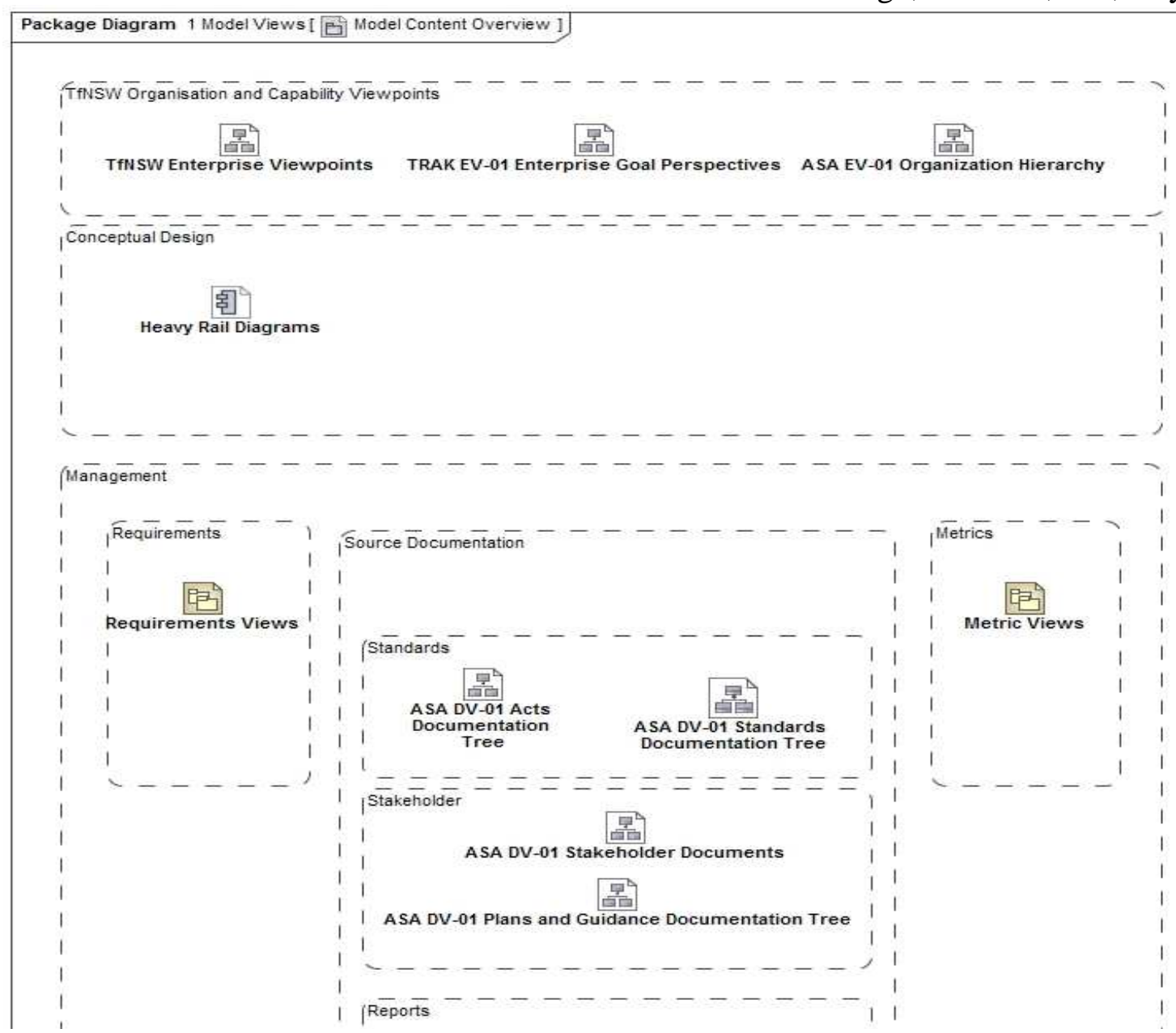

Figure 2: Package Diagram used as an overview of the Framework Structure

\section{Enterprise-level Modelling for Transport System Drivers}

One area of the framework pertains to identification of the needs of the various organisations and how these influence the acquisition and operation of the transport system. As previously indicated multiple enterprises contribute to the goals of TfNSW in supplying an updated and improved transportation experience for the commuters. Complicating the situation is the interdependencies between these enterprises. Some enterprises contribute to the goals of other organisations and so a representation of this complexity is needed to clarify what are the needs of a particular system under development based on the competing needs of the owning organisations.

Therefore views are created for each enterprise that identifies each enterprise's goals and how these goals are decomposed into needed capabilities to be provided within the transport system. An additional view has been created specifically for the inter-relation between the various enterprise needs.

The following diagrams show some common diagram structures. The enterprise-level modelling begins with the identification of the various enterprises that participate in the development, maintenance and operation of the transport system. Figure 3 provides an example of how the enterprises can be both identified and have their interrelationship specified. Understanding and being able to access the details of these interrelationships is very important because many enterprises contribute to the goals of higher level enterprises. 


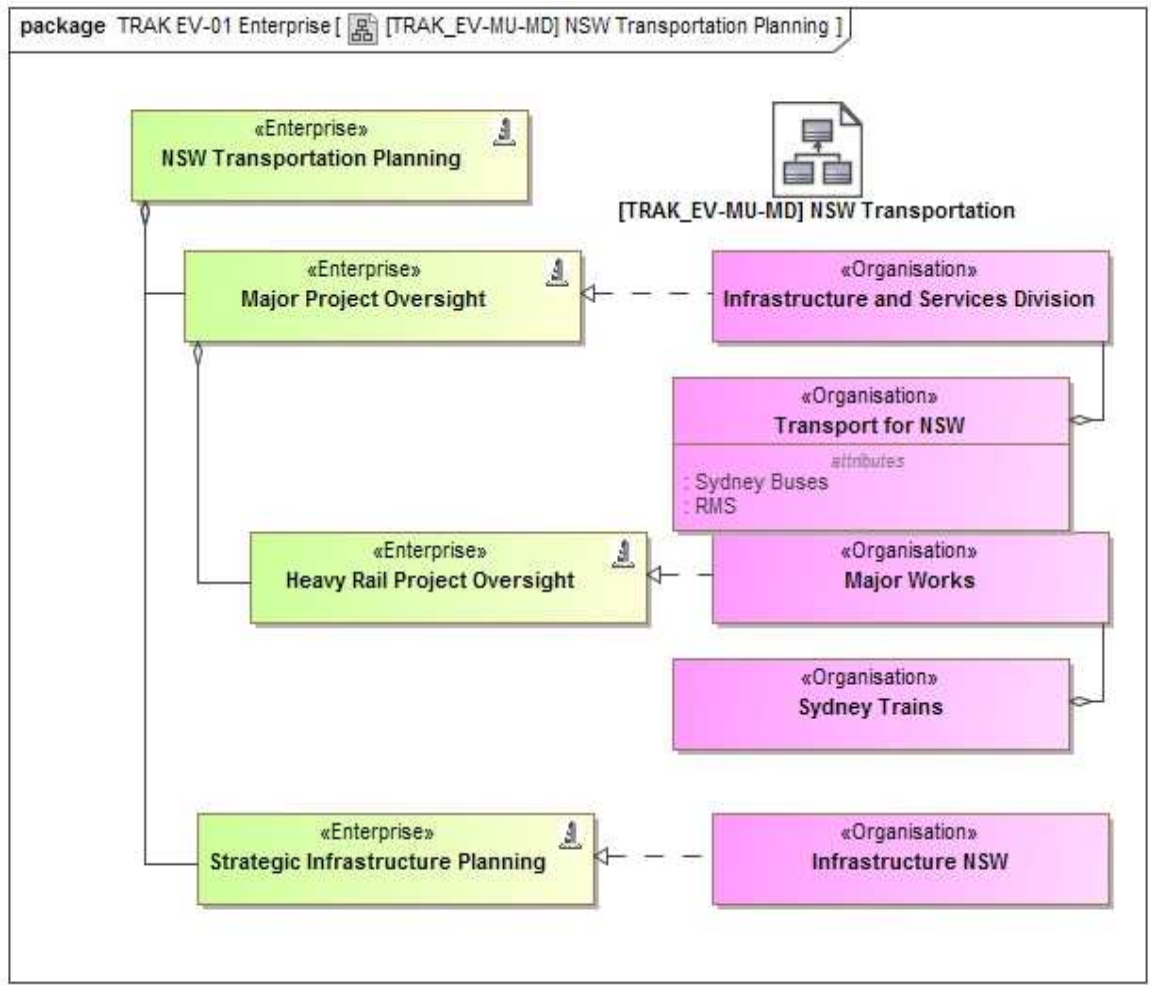

Figure 3: Class diagram used to identify the Enterprise relationships

As a result of these interrelationships and dependencies it is necessary to articulate the goals of the various enterprises. Figure 4 depicts the derivation of the goals of a particular enterprise, in this case NSW Heavy Rail Transport. In the figure there are a number of enterprise goals that have been further decomposed to ascertain the capabilities required to meet these goals. The figure also shows how it is possible to relate metrics to these capabilities. Articulation in this manner is important as it is necessary to monitor the evolving needs of the system. While the goals for an enterprise may not change at the higher level, the levels of capability may change over time as greater demands are placed on the system. Therefore it is necessary to provide these relationships so the evolution of these demands can be articulated and managed through future acquisition activities understanding the appropriate levels of demand. 


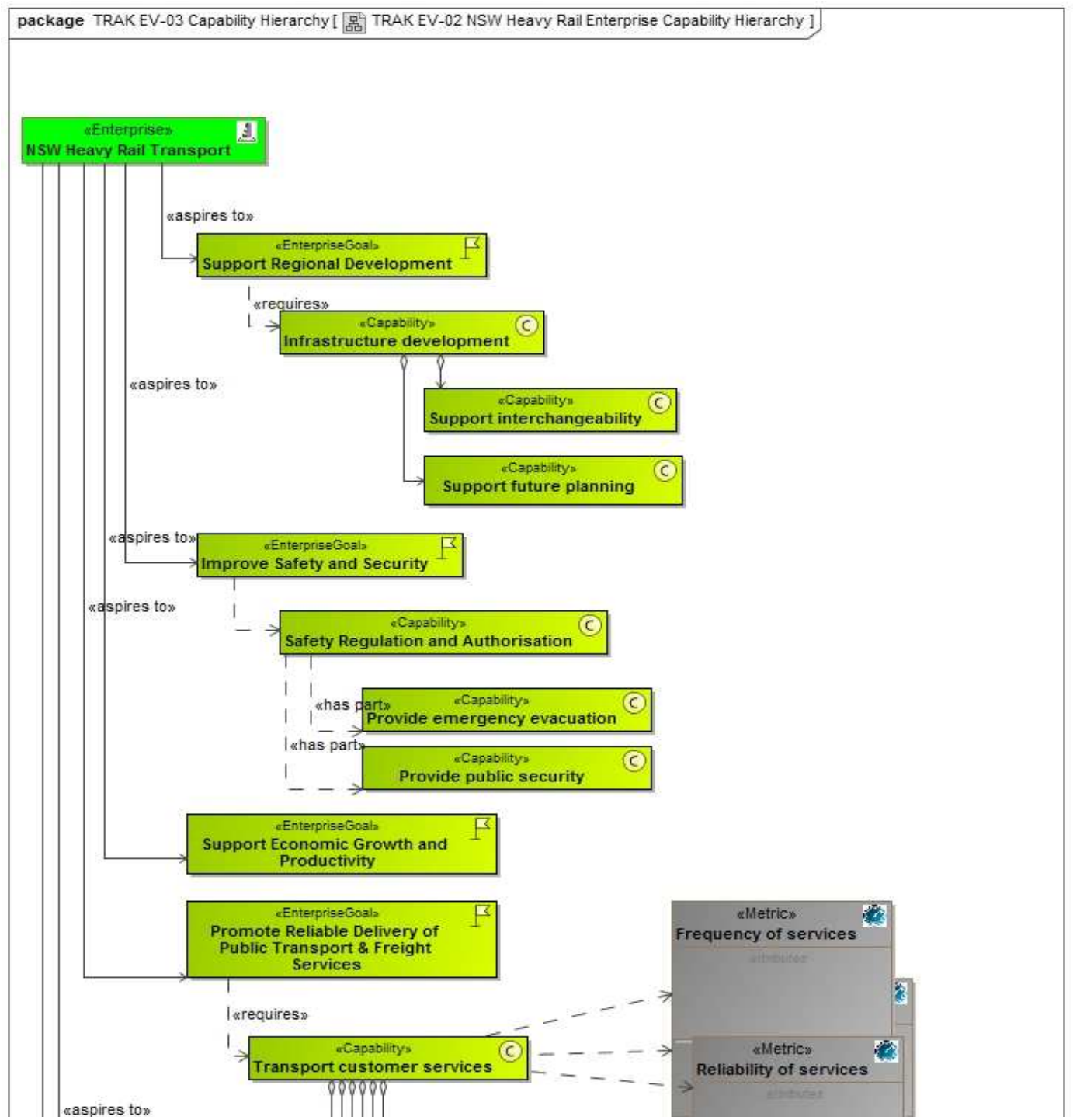

Figure 4: Class diagram used to capture the derivation of Enterprise Goals and Metrics

The various enterprises can also be decomposed to identify the personnel that comprise them as shown in Figure 5. The ability to capture the various positions enables the depiction of the roles that they play in the organization responsible for the transport system (captured in views not presented here for brevity). These roles are mapped onto the activities that they perform in various contexts (see section below on behavior modelling in the contextual design). This depiction can be used to redistribute roles or functions when the organization is modified or transferred between human and systems. This information is critical to ensure that safety is not compromised by understanding what element (system or person) undertakes the roles required to ensure safety. These elements can then be tested or trained respectively to ensure that they can undertake the activities necessary to fulfil these roles. This line of inquiry may also result in advice of necessary training when introducing new arrangements or systems. 


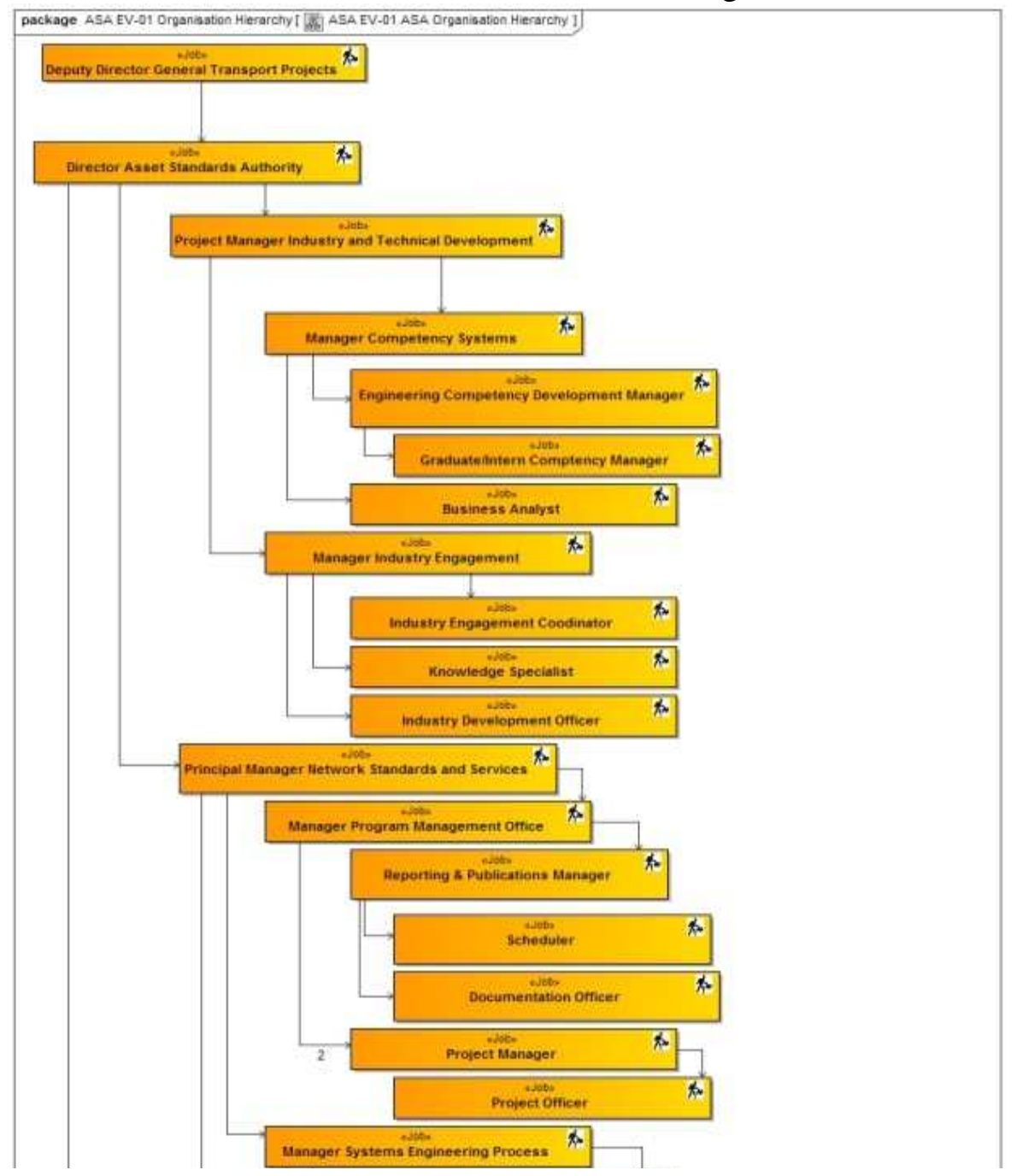

Figure 5: Class diagram used to capture the organization hierarchy

\section{Document and Existing Transport System Drivers}

While the enterprise goals provide top-down drivers for the transport system, there are bottom up drivers based on the fact that the transport system exists, but is also undergoing evolution. These bottom-up drivers include the operational concept, the existing infrastructure that will need to be interfaced to, and standards that are to be adhered to.

Although some of these diagrams (such as Figures 6 and 7) are often impossible to read on the page of a document this is not an issue in using the framework as they are designed as entry points into the framework and its database rather than only being guides to enterprise structure. The framework is designed and expected to only be used on a capable computer system where such diagrams can always be expanded so that the diagrams can be read.

\section{Conceptual Architecture of the Existing Transport System}

The conceptual architecture specifies how the system currently operates so that understanding can be made of how the current operations will change as new systems are introduced. After a new infrastructure project has been delivered, this will often cause changes in the operational concept which need to be identified for assurance and to ensure proper training is undertaken. The conceptual architecture is divided into conceptual Physical depictions, Behavioural modelling, and Standards and Documentation. Each of these is elaborated below. 
Conceptual Physical Depictions. The development of the physical region of the concept design has allowed several alternative depictions of the same information. The physical region is intended to identify the sub-elements that comprise particular types of elements. SysML enables us to present this information in a variety of representations as depicted in Figure 6. This figure depicts the top level elements that comprise a passenger exchange but in two different orientations. At the top is a hierarchical presentation of the elements below which are the same elements depicted as an internal block diagram (IBD). The hierarchical diagram quickly conveys the elements that have been identified and allows the presentation of information down several levels. The IBD representation has been created to depict the various paths a commuter may utilise to pass through the station. This is more complex but provides more structure about the assembly.
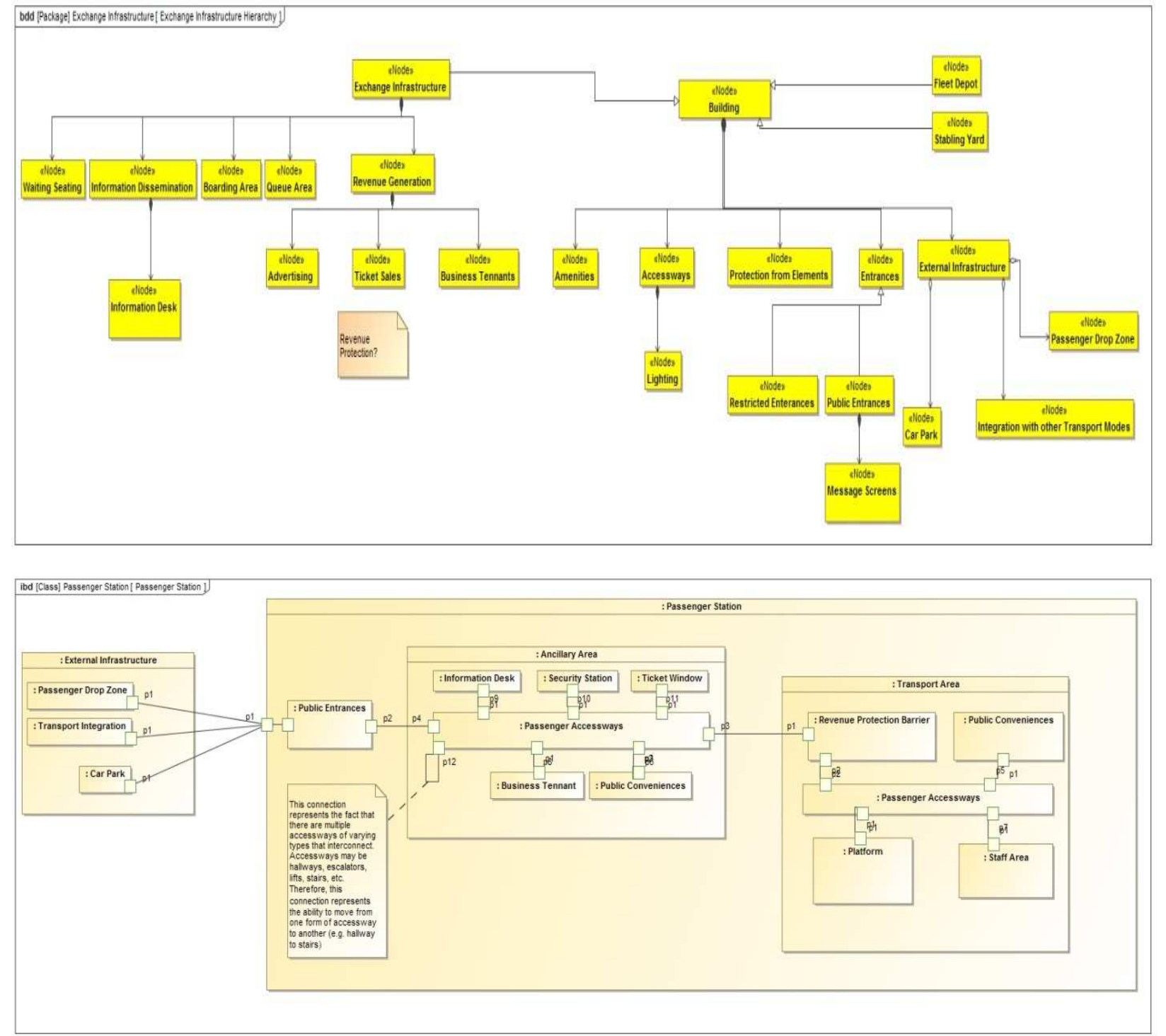

Figure 6: Alternate views of physical system elements: Block definition diagram (top) and internal block definition diagram (bottom)

Behavioural Representations. The behavioural region of the conceptual design is dedicated to articulating the functions and how they combine to yield the desired behaviour. Functionality can be presented in a hierarchical format similar to the physical (seen in the top Figure 6) or it can be presented in one of several formats depicted in Figure 7. On the left can be seen an activity diagram of a type that is used for high level processes that are largely the responsibility of a single element. On the right of Figure 7 can be seen a swimlane diagram that shows the complex interaction needed to control rolling stock (i.e. trains) so that they safely move along a clear track. Note that this is a 
$26^{\text {th }}$ Annual INCOSE International Symposium (IS 2016)

Edinburgh, Scotland, UK, July 18-21, 2016

conceptual design so it is solution independent and genericised so that it is applicable to the current context, fast trains (where trackside signage is not viable) or alternatively fully automated trains.

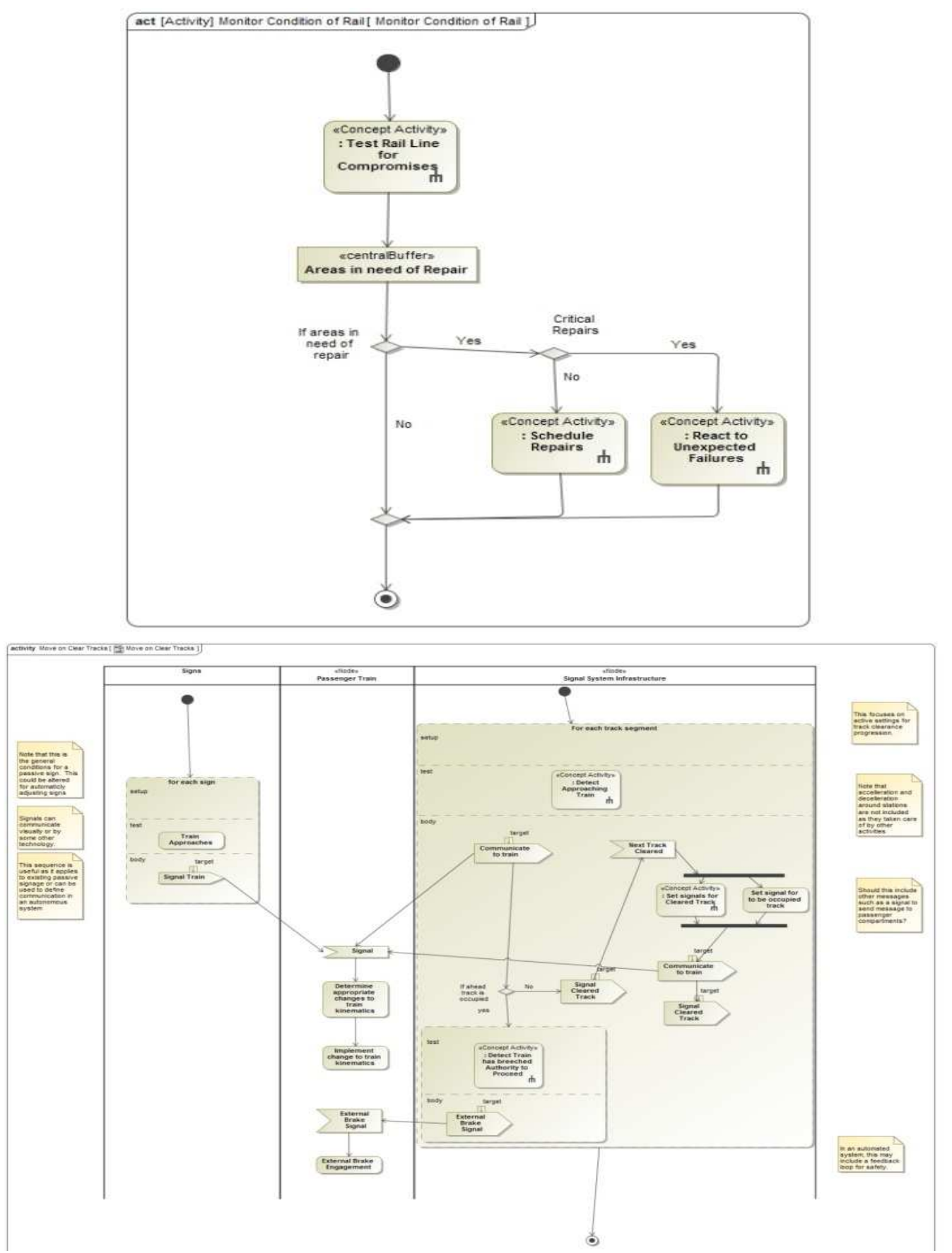

Figure 7: Behavior modelling examples: Activity diagram (top) and swimlane diagram (bottom)

The swimlane diagram has the additional benefit of indicating the elements that have been assigned to perform the functions. This element could be a physical element that will be implemented by a system or it could be a role performed as a human. This is very potent as it both shows how humans are directly interacting with their systems, but also allows the transfer of functions between humans and systems as the transport system evolves. This transfer can be seen in the changes in such diagrams and therefore the evolving roles of humans as well as the systems they utilize. 


\section{Standards and Documentation}

Many parts of the transport system are also constrained by standards and similar documentation (such as parliamentary acts or internal policies). We will use the term standard in this section to apply to the collective group. Management of documentation becomes complicated as a result of cross-referral and referencing. While many new standards are attempting to minimise this trend, the problem will persist for some time into the future. Therefore it is important to be able to understand not only how a particular standard affects elements within the system but the interdependencies between these standards. Figure 8 shows an example of how the interrelationships between the documents can be articulated for ease of access and to show the relationships between them. Here you can see that the document at the top of the diagram requires a number of other documents. Taking this relationship to another level and we begin to see the complex cross-referencing between documents that can be difficult to manage, when thousands of documents are involved.

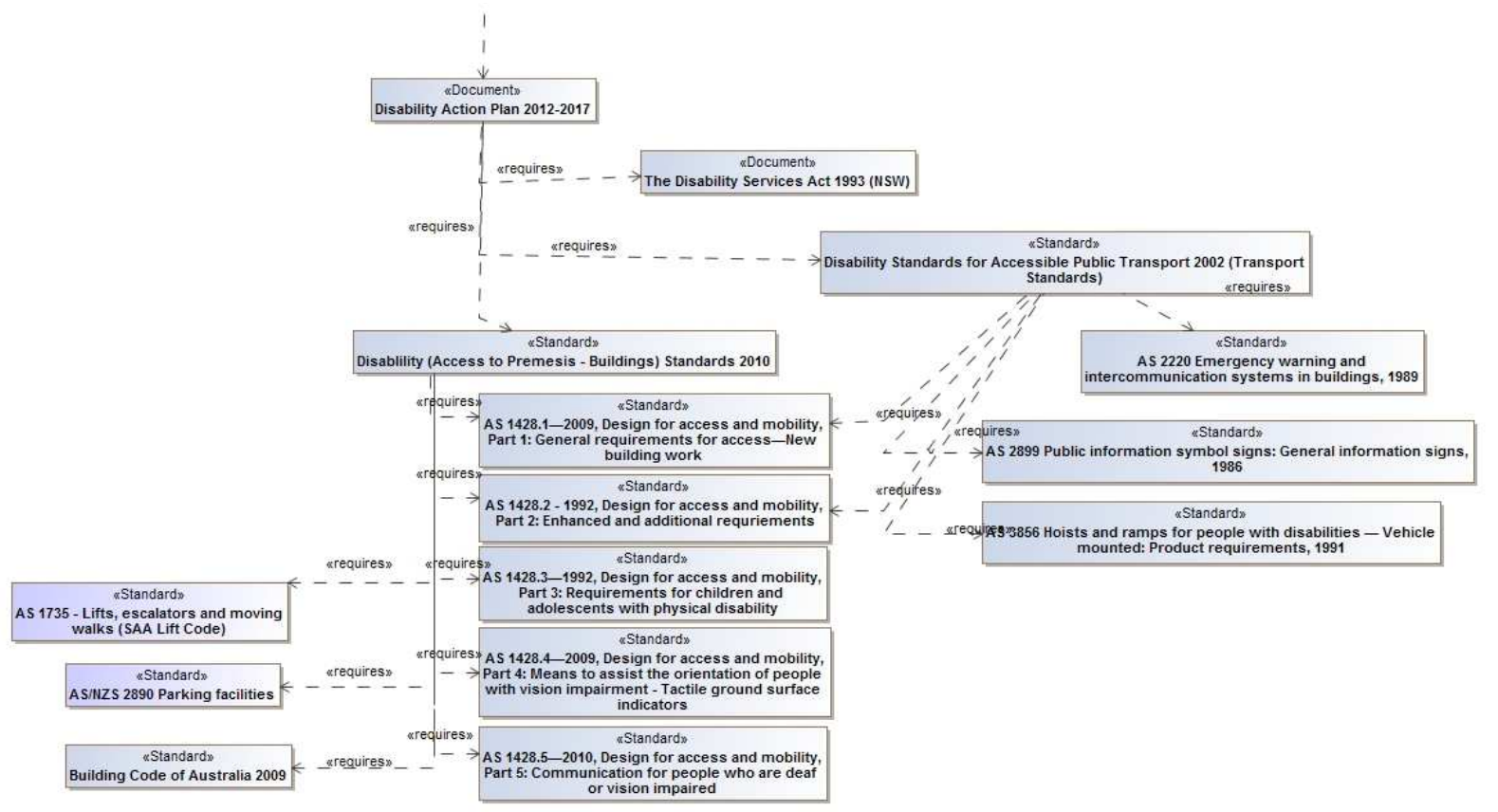

Figure 8: Class diagram excerpt to illustrate capture of document inter-relationships

Figure 9 provides examples of how document information can be related to the rest of the framework. On the left is a diagram that articulates the content of a requirement document while preserving the document's structure. In yellow is the heading hierarchy while the requirements are shown in pink. The diagram has also been able to preserve figures from the document that are referenced, which is directly useful as they provide concise information referenced by the text. While this example is from a requirements specification, the same type of diagrammatic interface to information can be done from any document that is referenced. For example, strategy documents have been processed to identify enterprise goals, capabilities and metrics. This type of depiction is highly useful as it facilitates identification of where data came from and enables the data to be seen in context of the original source. An additional benefit of this type of diagram is that it can help identify changes in documentation by direct comparison of the document trees. 


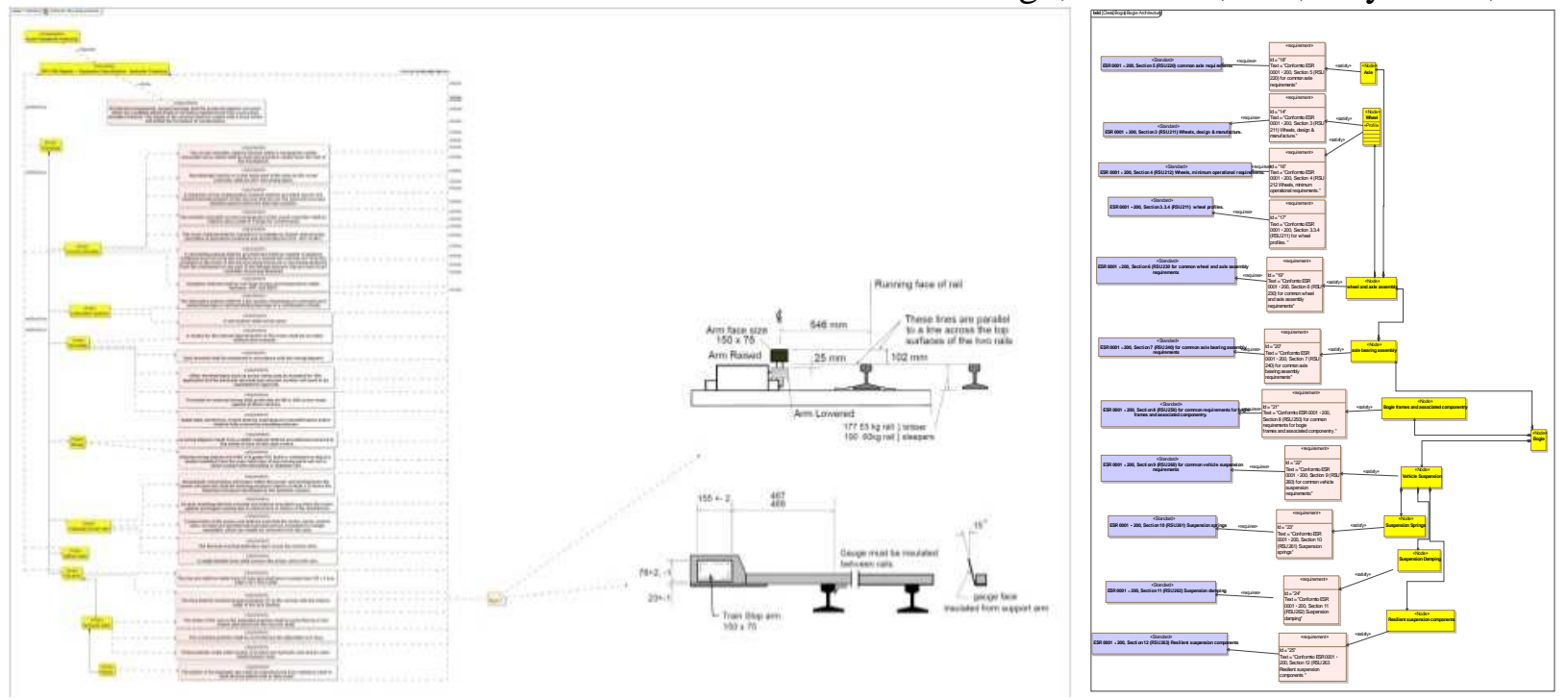

Figure 9: Example class diagrams depicting relation of information to and from a document

On the right of Figure 9 is an example of how elements can have references summarized in a single diagram. In the figure are the assembly components in yellow for a bogie (the undercarriage of rolling stock) with many of the sub-components having requirements that reference standards. Therefore the diagram depicts the element, the requirement (in pink) that specifies the standard to which compliance is necessary and then the document object. In Magic Draw, objects can be linked to URLs or documents so this diagram can be used to make many of the standards that need to be met easily accessible. Also, the two diagrams together make a powerful depiction as the right diagram defines the standards that have to be met and a diagram of the left style can be constructed for each standard that articulates the content and confirms the content in the database. Although this diagram (Figures 9) and others are often impossible to read on the page of a document this is not an issue in using the model as they are designed as entry points into the model and its database rather than only being guides to enterprise structure. The model is designed and expected to only be used on a capable computer system where such diagrams can always be blown up so that the diagrams can be read.

\section{Development of a Generic Transport System Framework}

A generic framework of a transport system has then been developed and related back to the enterprise-level and existing information. This region within the TNAF is designed to capture:

- the conceptual physical architecture

- interfaces

- behaviour and

- interactions

within a generic transport system. This representation is intended to be not only solution independent but also facilitate future configurations and technologies. Therefore the generic framework is intended to support the changes of assignment of activities necessary between people and systems to depict the future evolution from human-centric activities to autonomous systems with human oversight. This transition is a major concern to all heavy rail organisations at present, but has implications for other surface transport sectors as well. Although it has been recognised that it will be extremely useful addition to the framework, we have not so far been in a position to include consideration of the availability and sustainability of funding for the very large projects with which TfNSW is faced.

The framework also provides the ability to capture how different modes of transport interface, so that more seamless transitions between modes can be provided for commuters. TfNSW has stated 
that it wishes to form a more integrated, multimodal transport system (TfNSW 2012) but many of the existing transport modes focus on their own purview. This ability to capture how the modes of transport interconnect is intended to yield an overall better understanding of how the commuter will move from "door to destination".

To assist in the understanding of the interoperations of the system, the framework has also been designed to capture multiple perspectives of the system. As a perspective is a view into the framework, the ability to capture alternative perspectives of the same interaction is a valuable ability. This allows each stakeholder to understand their purview and understand how changes for one stakeholder impact other stakeholders.

\section{System Evolution for Safety Assurance}

One of the challenges with projects which change existing infrastructure is that the transition time for the changes to take place can be considerable. For example, a heavy rail upgrade will be rolled out line by line which can causes safety assurance challenges as the configuration of the various lines change over time. This can be further compounded if there are parallel rollouts from several projects simultaneously.

To manage for this, the operational concepts have to be evolved from the existing situation. The various projects need then to be examined to identify the milestones that make changes to the existing transport system. These milestones are mapped onto the network to identify when particular changes are made to particular areas of the transport system. The existing operational concepts can then be evolved for the changing situations as the transport system evolves and the safety assurance can then be made against each of these operational concepts. This results in very good safety assurance through the delivery of transport system upgrades and beyond.

\section{Conclusion}

The development and application of the TNAF has proven to be an effective means to get stakeholders to communicate through a common depiction of the system structure. The ability to model an interaction from multiple perspectives has also enabled the integration of the information needs of the different stakeholders into dedicated, interrelated views which are now being used to influence how TfNSW organizations depict the current and future operation of the transport system as well as the constraints (such as standards and regulations) that have to be met during acquisition.

\section{References}

ASA 2014 Network Standards Numbering System T MU MD 00001 SP Accessed Online at http://www.asa.transport.nsw.gov.au/sites/default/files/asa/asa-standards/t-mu-md-00001sp.pdf

IEEE Std 1471, IEEE Recommended Practice for Architectural Description of Software Intensive Systems. 2000.

International Organization for Standardization/International Electrotechnical Commission, ISO/IEC 15288:2002, Systems Engineering - System Life Cycle Processes. Nov. 15, 2003.

ISO/IEC/IEEE 42010, Systems and software engineering — Architecture description. 2011.

Matthew Hause An Overview of UPDM - A Unified Profile for DoDAF/MODAF. 2009.

Matthew Hause, Model- Based System of Systems Engineering with UPDM. International council on Systems Engineering (INCOSE), 2010.

Nic Plum, An Implementation of TRAK in Sparx Systems Enterprise Architect in INCOSE UK chapter's Annual Systems Engineering Conference. 2010. 
$26^{\text {th }}$ Annual INCOSE International Symposium (IS 2016)

Edinburgh, Scotland, UK, July 18-21, 2016

Nic Plum. TRAK. 2012 10/8/2012]; Available from: http://trakcommunity.org/index.php/wiki/TRAK.

Object Management Group, OMG Unified Modeling Language (OMG UML) Infrastructure Specification, v2.3. May 2010a: http://www.omg.org/spec/UML/2.3/Infrastructure.

Object Management Group, SysML OMG Specification. 2010b: http://www.omgsysml.org.

TfNSW 2012 NSW long Term $\quad$ Master Plan

http://www.transport.nsw.gov.au/sites/default/files/b2b/publications/nsw-transportmasterplan-final.pdf

UK Ministry of Defence, MODAF M3 version 1.2.004, Defence, Editor. 2013.

U.S. Department of Defense, DoDAF Architecture Framework Version 2.02, Architecture Framework Working Group, Editor. 2010.

\section{Biography}

William Scott has been engaged in Systems Engineering since the 90s where he undertook research at the University of South Australia into enhancing SE tool capabilities using artificial intelligence techniques. Since then he has been engaged in modelling and simulation activities that aim to enhance SE activities largely in Australian defence but also other areas such as hospital operations.

Since he moved to University of Wollongong, he has been engaged in examining the application of MBSE to assist the acquisition and modelling of public transport system for TfNSW and Australian heavy rail. These activities have involved a combination of development of modelling practice based on world best practice, examination of application to increase usability and utility of AF tools, and to develop enhanced, customized functionality to automate common activities, increase usability, and reduce effort.

Gary Arabian is an enthusiastic and proactive engineer who likes to solve complex problems with a passion. After completing a Bachelor of Engineering in Civil Engineering at University of Technology, Sydney (UTS) in 2012, and working for a few years in different engineering industries in multiple roles, he felt the need to further his knowledge in engineering management. In 2014, he completed his Master of Engineering Management at UTS. Since then he has been a member of the systems engineering team at the Asset Standards Authority (a branch of Transport for NSW). During his time with the ASA, he has helped communicate Model Based Systems Engineering across the Transport cluster and managed the development of the Transport Network Functional Architecture.

Richard Fullalove is an engineering professional with 30 years' experience, involving research \& development, progressing to signal design management, construction, testing, commissioning and maintenance. He's worked on capital projects for 20 years, of which the last 15 years focused on multi-disciplinary rail systems engineering (SE), integration, system safety and assurance.

Over his career he's held various senior engineering management positions including Regional Signal Manager, Project Manager, Principal Engineer and Systems Engineering Manager, focusing on innovation, process improvement, interface and integration, systematic engineering solutions and new capital projects.

His experience has evolved beyond signalling to engineering and integration of rail systems, including signalling, telecommunications, control systems, electrical traction (AC \& DC), overhead wiring systems, high voltage feeders, track, buildings \& structures, earthworks, and station systems. He's managed or carried out requirements engineering, systems architecture development, interface management, human factors integration, RAM management, EMC management, systems safety 
$26^{\text {th }}$ Annual INCOSE International Symposium (IS 2016)

Edinburgh, Scotland, UK, July 18-21, 2016

assurance, verification, validation and operational readiness planning. He is experienced in heavy rail passenger, rapid transit metro, semi-high speed passenger, general freight, and heavy haul rail systems and operations. He's also gained experience on major programs such as the West Coast Route Modernization (UK), Manchester South Capacity Improvement Program (UK), Victoria Line Upgrade Program (UK), Epping-Chatswood Rail Link (Australia) and Novo Rail Alliance (Australia).

Richard holds a Master's Degree in Electrical Engineering, Chartered Professional Engineer, and on the National Professional Engineers Register (NPER).

Peter Campbell is an Honorary Research Professor at the University of Wollongong and recently retired from the Defence and Systems Institute at the University of South Australia where he was engaged in the use of MBSE in defence and infrastructure applications over the last 10 years. Prior to his university appointments he was a research centre leader and the director of the Decision and Information Sciences Division at Argonne National Laboratory (now renamed Global Security Sciences) which specialises in the development of decision support tools and analysis for application to complex behavioural problems for US government agencies. He is currently the director of several projects to develop architecture framework models for the rail industry for UOW, and more broadly to assist in the management and delivery of the complex of transport infrastructure projects that Transport for NSW, Australia is currently engaged in delivering. Peter has been a member of INCOSE since 2009 and participates in the Infrastructure, CIPR and MBSE working groups. 